J. Appl. Cryst. (1974). 7, 189

\title{
Study of Polymer Solution by Small-Angle Neutron Scattering in the Intermediate Momentum Range
}

\author{
By J. P. Cotton, B. Farnoux, G. Jannink and R. ObeR* \\ Service de Physique du Solide et de Rèsonance Magnètique, Centre d'Etudes Nucléaires de Saclay, BP $n^{\circ} 2-91190$, \\ Gif-sur-Yvette, France
}

In the investigation of polymer solutions by small-angle neutron or X-ray scattering, the intermediate momenutm range corresponds to the distance between the radius of gyration $R_{G}$ 'and the statistical subunit length $l$ of the polymer chain. In this range, where the specific polymeric behaviour prevails, it can be shown, using the Debye relation, that the scattering curve presents a $q^{-2}$ singularity, $q$ being the momentum transfer. For real chains in solution this singularity is broadened by different factors: 1 - For a vanishing concentration $c$, there is a trivial effect due to the finite mass of the polymer [H. Benoit (1953). J. Polym. Sci., XI (5), 507-510]. The scattering law is written as

$$
\underset{c \rightarrow 0}{S(q)}=\frac{k c}{R_{G}^{2}\left(q^{2}+\frac{M_{w}}{M_{n}} R_{G}^{-2}\right)}
$$

where $k$ is constant, $M_{w}$ and $M_{n}$ are respectively the weight-average and the number-average molecular weight. 2 - For non-zero concentrations another broadening appears, due to the pair interaction between molecules. The scattering law is now

$$
S(q)=\frac{k c}{R_{G}^{2}\left(q^{2}+\frac{M_{w}}{M_{n}} R_{G}^{-2}+\xi_{(c)}^{-2}\right)} .
$$

The pair interaction term $\xi^{-2}(c)$ is linearly dependent on concentration. When the concentration is high enough one expects deviation of this broadening from linearity due to triplet interaction, etc. However in the case of a polymer solution a characteristic effect is predicted. Two concentration ranges are defined with respect to a critical concentration $C^{*}$ at which the distance between chains $d$ equals the radius of gyration $R_{G}$. $(A)$ For $C>C^{*}$, but well below the density of bulk material, the chains overlap, and $\xi^{-2}(c)$ represents the pair interaction between segments. The inequality $d<R_{G}$ and the Flory inequality $v N^{1 / 2}>l^{3}$, where $N$ is the number of segments per chain, indicate that $R_{G}^{2} \xi^{-2}>1$. Thus the scattering law reduces to the simple Lorentzian form

$$
S(q)=\frac{k c}{R_{G}^{2}\left(q^{2}+\xi_{(c)}^{-2}\right)}
$$

where the term $\xi^{-2}(c)$ is interpreted as a screening length and is given by

$$
\xi_{(c)}^{2}=\xi_{s d}^{-2}=A v c
$$

where $A$ is a constant independent of molecular mass, and $v$ the excluded volume, for one statistical unit of the chain. Evidence for the 'semi-dilute' range of polymer solutions must be given by the $c$ dependence of $\xi^{-2}$. This has been done by small-angle neutron scattering [J. P. Cotton, B. Farnoux \& G. Jannink (1972).J. Chem. Phys. 57, 290-294] for polystyrene of molecular mass $M_{1}=6 \cdot 5 \times 10^{5}$ and $M_{2}=2 \cdot 1 \times 10^{6}$ in solution in deuterated benzene. $(B)$ For $C>C^{*}$ the solution is dilute and the broadening $\xi^{-2}(c)$ takes into account the pair interaction between the chains. The chain concentration expansion of the thermodynamical functions yields

$$
\xi_{(c)}^{-2}=\xi_{d}^{-2}=A\left(u / N^{2}\right) c
$$

where $u$ is the excluded volume for the coil-coil interaction. Experimental values of $A$ and $u$ indicate that $R_{G}^{2} \xi_{d}^{-2}>1$, for concentrations greater than $10^{-3} \mathrm{~g} \mathrm{~cm}^{-3}$ and for polymer molecular masses greater than $10^{5}$. The Lorentzian form was thus again used to interpret the small-angle scattering experiment. The data where taken from a light scattering experiment with polystyrene of molecular mass $M_{3}=7 \cdot 5 \times$ $10^{6}$ in solution in benzene [A. Benoit \& C. Picot (1966). Pure Appl. Chem. 12, 545-561]. Interpolation of the light scattering and neutron scattering data gives a continuous concentration dependence of $\xi^{-2}$ between $10^{-3}$ and $10^{-1} \mathrm{~g} \mathrm{~cm}^{-3}$ and shows: (1) $\xi^{-2}$ is linearly dependent upon $c$ above $C^{*}$ and below $C^{*},(2)$ around $C^{*} \xi^{-2}$ deviates from linearity; (3) $\xi_{s d}<\xi_{d}$, which reflects the inequality $u<N^{2} v$. This last inequality is noteworthy as a typical polymer configuration effect. Osmotic pressure concentration dependence agrees also with this interpretation [J. P. Cotton, B. Farnoux, G. Jannink \& R. Ober (1973). J. Phys. A: Math. Nucl. Gen. 6, 951-957; J. P. Cotton, B. Farnoux, G. Jannink \& C. Strazielle (1974). J. Polym. Sci. C. In the press].

* Laboratoire de la Matière Condensée, Collège de France, 75005 Paris, France. 